\title{
Translation of Common Information Model to Web Ontology Language
}

\author{
Marta Majewska ${ }^{1}$, Bartosz Kryza ${ }^{2}$, and Jacek Kitowski ${ }^{1,2}$ \\ ${ }^{1}$ Institute of Computer Science AGH-UST, Mickiewicza 30, 30-059 Krakow, Poland \\ ${ }^{2}$ Academic Computer Centre Cyfronet-AGH, Nawojki 11, 30-950 Krakow, Poland \\ \{mmajew, bkryza, kito\}@agh.edu.pl
}

\begin{abstract}
This paper presents a brief overview of the work on translation of Common Information Model (CIM) to Web Ontology Language (OWL) standard. The main motivation for the work is given, along with discussion of major issues faced during this work. The paper contains also comparison of existing approaches to conversion of CIM to OWL and presents the CIM2OWL tool that performs the conversion of CIM schema and allows convertion of CIM instances - representing for instance configurations of particular systems - to OWL individuals.
\end{abstract}

Key words: Metadata, ontologies, Grid computing, Common Information Model, ontology translation.

\section{Introduction}

Several researchers have risen lately the issue of translation of existing and widely recognized Distributed Management Task Force (DMTF) standard for resource description called Common Information Model (CIM) 1 to Web Ontology Language (OWL) 2]. Especially in the Grid setting, where OWL could be used for representation of semantic Grid metadata, the problem of the interoperability appears. Among reference ontologies for modeling the hardware and software computer resources the DMTF Common Information Model as well known, organizationally supported and regularly updated meta-model for the considered area (e.g. popularly referred in software for management of systems, networks, users and applications across multiple vendor environments) seemed promising. CIM is a hybrid approach, inspired by the object oriented modeling and database information modeling. The CIM Schema consists in particular of Core and Common Models as well as developed by users Extension Schemas. As it introduces the metadata for annotating model classes and instances, it is partially not compliant with the UML methodology. OWL is W3C recommended ontology language for the Semantic Web, which exploits many of the strengths of Description Logics, including well defined semantics and practical reasoning techniques. OWL offers greater expressiveness of information content description then that supported by XML, RDF, and RDF Schema, by providing additional vocabulary along with some formal semantics. 


\section{CIM to OWL Mapping}

In the beginning, the mapping between semantically equivalent constructs in MOF and OWL was established. That included class definition, inheritance (to some extent), data type attributes, cardinality constraints, comments, etc. The succeeding step in definition of the mapping was to extend the existing mapping with representations of MOF constructs, which do not have direct equivalents in OWL.

Table 1. The mapping definition from CIM to OWL

\begin{tabular}{|c|c|}
\hline CIM Artifact & OWL Construct \\
\hline Class & 〈owl:Class> \\
\hline Generalization & $\langle\mathrm{rdfs}$ : subClassOf $\rangle$ \\
\hline $\begin{array}{l}\text { Association } \\
\text { (Aggregation, } \\
\text { Composition) }\end{array}$ & $\begin{array}{l}\text { <owl: } \text { Class rdf:ID="..."> } \\
\quad<\text { rdfs: subClassOf } \\
\quad \text { rdf:resource="cim-meta:CIM_Association/> } \\
\text { </owl:Class }>\end{array}$ \\
\hline Property & <owl:DatatypeProperty> \\
\hline REF Property & <owl:ObjectProperty> \\
\hline Method & <cim-meta: hasMethod> \\
\hline Default Value & 〈cim-meta:defaultValue > \\
\hline Override & $\langle$ rdfs:subPropertyOf $\rangle$ \\
\hline Key & <owl: InverseFunctionalProperty> \\
\hline Min, Max & 〈owl:minCardinality>, <owl:maxCardinality> \\
\hline ValueMap, Values & $\begin{array}{l}\text { <cim-meta:CIM_Value> composed of <cim-meta:value> } \\
\text { and <cim-meta:valueMap> }\end{array}$ \\
\hline Deprecated & 〈owl: deprecatedClass> or 〈owl: deprecatedProperty> \\
\hline Required & $\begin{array}{l}\text { <owl:minCardinality rdf:datatype="\&xsd;int">1 } \\
\text { </owl:minCardinality> }\end{array}$ \\
\hline Experimental & <cim-meta:Experimental> \\
\hline Alias & $\begin{array}{l}\text { <owl: equivalentClass>, <owl: equivalentProperty> } \\
\text { or <owl: sameAs> }\end{array}$ \\
\hline ModelCorrespondence & $\langle$ rdfs:seeAlso $\rangle$ \\
\hline Read, Write & 〈cim-meta:readable>, 〈cim-meta:writeable> \\
\hline Version & <cim-meta: cimVersion> \\
\hline Abstract & <cim-meta: Abstract> \\
\hline Units & $\langle r$ df $\mathrm{s}:$ comment $\rangle$ \\
\hline Vectors & $\langle$ rdfs: comment $\rangle$ \\
\hline
\end{tabular}

The lack of fully equivalent constructions or rules for expressing some MOF constructs in OWL implied that partial, simplified mappings had to be admitted. These unambiguous mappings concerned mainly data restrictions (e.g. qualifier Value, ValueMaps), distinctions (e.g. qualifier Key, Propagated, Weak), redefinitions (e.g. qualifier Override), access (e.g. qualifier Read, Write), versioning (qualifier Version), default values, abstracting (qualifier Abstract), and dynamics (e.g. procedures, qualifier IN, OUT). Unfortunately, many issues of 
the not fully semantically equivalent part schema mapping are out of the scope of this paper. To achieve optimal mappings a few approaches were used such as usage of various semantic constructs with an approximate and uncontradictory meaning (e.g. for qualifier Override), definition of an additional meta ontology for a provision of missing CIM vocabulary called cim-meta (e.g. for qualifiers Abstract, ValueMap, Values, default values) and usage of annotation properties and comments (e.g. for qualifier Units). An excerpt from mapping is given in Table1(see http://gom.kwfgrid.net for details). The resulting OWL ontology has OWL DL expressiveness except for Key qualifier which requires InverseFunctionalProperty to be applied to datatype values, which is not allowed in OWL DL. Thus using our tool, the user can choose to create OWL Full ontology with the Key qualifier, or stay in OWL DL dialect without the Key property. The instance mapping is provided based on the schema mapping. An occurrence of various qualifiers in class definitions usually does not influence the conversion of MOF instances to OWL and definition of new instances based on the OWL ontology.

\section{Related Work}

In K-Wf Grid project, a set of ontologies describing generic and domain specific aspects of the Grid were gathered and integrated for the thematic areas of workflows, Grid applications, services, data and resources [3]. Ontologies stored and managed by the component called Grid Organizational Memory (GOM) 4] are optimized for use in the Grid applications [5. The authors of [6] propose to use XML-based ontology language to define resource management information for network management systems. In [7, authors present a generic and extensible ontology for describing several aspects of Grid environment. In [8] an idea of developing semantic descriptions of the web services based on the CIM specifications is presented. In [9] the mapping of CIM UML notation to RDF-S, and the extension to OWL, are presented. The author of [10] focuses on the troublesome CIM to OWL translation issues. Currently one translation tool is available called CIMTool [11. Several generic knowledge representation translators also exist such as those described in [12] or [13].

\section{Conclusions}

As a result of our work we have created the mapping schema from CIM to OWL, the OWL resource ontology based on CIM and the CIM2OWL tool, whose advantage is the ability to translate not only CIM schema (i.e. CIM Core and Common Models) but also CIM instances. The large scope of the expressiveness and functionality was transformed from CIM to OWL.

Acknowledgments. This reasearch has been done in the framework of EU IST2004-511385 K-Wf Grid project and EU-IST FP6-34363 Gredia project. AGH University of Science and Technology grant is also acknowledged. 


\section{References}

1. DMTF, Common Information Model (CIM) Standards, http://www.dmtf.org/standards/cim/.

2. W3C, Web Ontology Language (OWL), http://www.w3.org/2004/OWL/.

3. Kryza, B., Pieczykolan, J., Babik, M., Majewska, M., Slota, R., Hluchy, L. and Kitowski, J. Managing Semantic Metadata in K-Wf Grid with Grid Organizational Memory. In: Bubak, M. et al. (eds), Proc. of the 5th Cracow Grid Workshop (CGW05), Krakow, Poland, ACC-CYFRONET AGH, 2006, pp. 66-73.

4. Kryza, B., Pieczykolan. J and Kitowski. J: Grid Organizational Memory - A Versatile Solution for Ontology Management in the Grid, In Proc. of 2nd Intl. Conf. on e-Science and Grid Computing, Dec. 4-6, 2006, Amsterdam, (C) IEEE Computer Society Press, ISBN 0-7695-2734-5.

5. Kryza, B., Slota, R., Majewska, M., Pieczykolan, J., Kitowski, J. Grid Organizational Memory - Provision of a High-Level Grid Abstraction Layer Supported by Ontology Alignment. The Intnl Journal of FGCS, Grid Computing: Theory, methods \& Applications, vol. 23, issue 3, Mar 2007, Elsevier, 2007, pp. 348-358.

6. Lopez de Vergare, J.E., Villagra, V.A., Asensio, J.I., Berrocal, J. Application of the Web Ontology Language to define management information specifications. Proc. of the HP Openview University Association Workshop, France, 2004.

7. Xing, W., Dikaiakos, M. D., Sakellariou, R. A Core Grid Ontology for the Semantic Grid. In Proc. of 6th IEEE Intl. Sym. On Cluster Computing and the Grid (CCGRID'06) - Vol. 00, pp. 178-184. Washington, 2006

8. Keeney, J., Carey, K., Lewis, D., OSullivan, D., Wade, V. Ontology-based Semantics for Composable Autonomic Elements. Workshop of AI in Autonomic Communications at the 19th Intnl Joint Conf. on AI, Edinburgh, 2005.

9. Quirolgico, S., Assis, P., Westerinen, A., Baskey, M., Stokes, E. Toward a Formal Common Information Model Ontology. Proc. of Workshop on Intelligent Networked and Mobile Systems, WISE04, Australia, 2004, Springer, LNCS vol. 3307, pp. 1121.

10. Heimbigner, D. DMTF CIM to OWL: A Case Study in Ontology Conversion. In: Maurer, F. and Ruhe, R. (eds), Proc. of 16th. Intl. Conf. on Software Engng. and Knowledge Engng. (SEKE2004), Canada, 2004, Knowledge Systems Institute, Skokie, pp. 470-474.

11. CIMTool project website, http://cimtool.org/.

12. Corcho, O., Gomez-Perez, A. ODEDialect: a set of declarative languages for implementing ontology translation systems. ECAI2004 Workshop on Semantic Intelligent Middleware for the Web and the Grid. Valencia, Spain, 2004.

13. Chalupsky, H. OntoMorph: a translation system for symbolic knowledge. In A.G. Cohn, et al. (eds), editors, Principles of Knowledge Representation and Reasoning: Proc. of the 7th Intnl Conf. (KR2000), San Francisco, CA, 2000. Morgan Kaufmann. 